\title{
Bearing estimation using a large towed array
}

\author{
Melvin J. Hinich* and William Rule \\ The Virginia Polytechnic Institute and State University, Blacksburg, Virginia 24061
}

(Received 1 November 1974; revised 15 July 1975)

When a towed array of hydrophones is significantly nonlinear due to bending, ordinary linear array beamforming gives a biased estimate of the true source bearing. By processing the array as a sequence of smaller aperture subarrays and then computing the mean of the subarray bearings, the variation due to bending is reduced and a reasonably precise estimate is obtained if the average bending angle with respect to the nominal axis is small. The median and mean subarray bearings are analyzed for a theoretical statistical model and are tested using artificial data for various sinusoidal array geometries.

Subject Classification: $60.20 ; 30.82$.

\section{INTRODUCTION}

Towed hydrophone arrays have been extensively used in underwater acoustics research but have also been used to determine target bearing in certain military applications. When the tow ship is moving slowly on a straight line, the array is essentially linear and is usually steered by simple delay-and-sum beamforming, which has a left-right ambiguity since the array geometry is linear. This ambiguity is resolved by changing the heading of the tow ship. Changing direction and speed causes the array to bend. For nontrivial tow ship speeds, the array geometry has a snakelike pattern which varies over time during the transient (Fig. 1). This paper presents a signal-processing technique which mitigates the distortions due to array bending in the estimated bearing angle.

Consider a horizontal "linear" array of $K$ hydrophones which are electronically grouped into $J$ subarrays. Suppose that the small aperture subarrays of adjacent hydrophones have sufficient structural rigidity so that they are approximately linear. Assume that the vertical displacement of the hydrophones is in a constant density strata, and thus the array geometry can be treated as planar (two dimensional). By processing the array as a sequence of smaller aperture subarrays and then computing the mean subarray bearing, the variation due to bending is reduced and a reasonably precise estimate is obtained if the average inclination of the subarray is small.

Before going into the subarray-processing method, it is necessary to briefly review the statistical model used to develop the optimal array processor. Given a finite record of simultaneous observed sensor outputs, we wish to estimate the bearing of a farfield source of interest which is radiating energy at frequency $\omega_{0}$. In order to simplify the exposition, assume that the signal received at time $t$ and location $x$ on the array axis is a singlefrequency plane wave (Fig. 2):

$$
s(t, x)=A \exp \left[i\left(\omega_{0} t-\kappa_{0} x-\phi\right)\right],
$$

where $\kappa_{0}$ is the wavenumber. Letting $\theta$ denote the direction of propagation of the wave (the source bearing if the medium is horizontally homogenous), then for $0 \leqslant \theta$ $\leqslant \pi, \kappa_{0}=\left(\omega_{0} / v\right) \cos \theta$, where $v$ is the phase velocity. The parameters $A$ and $\phi$ are the amplitude and phase of the wave. For the general problem of a broad-band signal made up of a sum of overlapping plane waves, Hinich and Shaman ${ }^{1}$ analyze the maximum likelihood signal processing of a fixed array.

Suppose that we have a truly linear array. The output from the $k$ th sensor located at point $x_{k}$ is $p\left(t, x_{k}\right)=s\left(t, x_{k}\right)$ $+n\left(t, x_{k}\right)$, where $n\left(t, x_{k}\right)$ is assumed to be complex Gaussian noise whose variance, denoted $\sigma^{2}$, is the same at all sensors, i. e., the variances of the real and imaginary parts of the noise are equal to $\sigma^{2} / 2$. Given a $T \delta$ second discrete record of the array output (where $\delta$ denotes the sampling interval), the maximum likelihood estimator of $\theta$ is

$$
\hat{\theta}=\arccos \left(v \omega_{0}^{-1} \hat{\kappa}\right),
$$

where $\hat{\kappa}$ is the wavenumber which maximizes the frequency -wavenumber power spectrum

$$
S\left(\kappa, \omega_{0}\right)=K^{-1}\left|\sum_{t=1}^{T} \sum_{k=1}^{K} p\left(t, x_{k}\right) \exp \left[i\left(\kappa x_{k}-\omega_{0} t\right)\right]\right|^{2}
$$

If $\rho K$ is large, where $\rho=T A^{2} / \sigma^{2}$ is the signal-to-noise ratio in the $1 / T \delta \mathrm{Hz}$ band centered at $\omega_{0}$, then $\hat{\theta}$ is approximately Gaussian with mean $\theta$ and variance (in radians) $)^{2}$

$$
\sigma^{2}(\hat{\theta})=\frac{3}{2 K W^{2} \rho(\pi \sin \theta)^{2}},
$$

where $W$ is the aperture of the array as measured by the number of wavelengths, $\lambda=2 \pi v / \omega_{0}$, and $\theta \neq 0, \pi$.

This result holds for small $K$ if $\rho$ goes to infinity, ${ }^{3}$ but it is questionable whether the approximation is good when $\rho$ is moderate and $K$ small. However, as is shown in Appendix A, the inequality

$$
K>8 \rho^{-1}
$$

provides a useful heuristic to insure that there is sufficient sensor redundancy in an equally spaced array so that the wave will be detected with probability greater than 0.95.

For incoherent Gaussian noise, the maximum likelihood technique is equivalent to delay-and-sum steering of the array in the direction which maximizes the total power over the observation period, after filtering at $\omega_{0 .}{ }^{4}$

It would be useful here to present a numerical example. Suppose that each subarray has eight hydrophones 


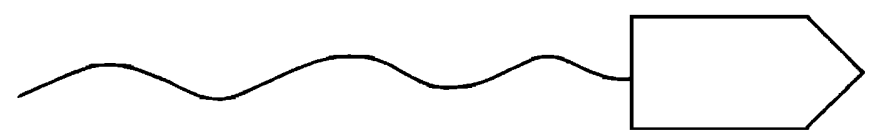

FIG. 1. Bent towed linear array.

which are $5 \mathrm{~m}$ apart. If the signal's frequency is $\mathbf{1 5 0}$ $\mathrm{Hz}$, the wavelength is $10 \mathrm{~m}$, using a speed of sound in the ocean of $1.5 \mathrm{~km} / \mathrm{sec}$, and thus $W=4$. Note that the sensors are spaced one-half wavelength apart, and thus there is no spatial aliasing.

If the channels are sampled at the Nyquist rate (every $3 \frac{1}{3} \mathrm{msec}$ ) for a $1-\mathrm{sec}$ period, then $T=300$. Consequently, if $\sigma=10 \mathrm{~A}$, then $\rho=3$ and the approximate rms error for $\hat{\theta}$ is then $(16 \pi|\sin \theta|)^{-1}$. Obviously, $K=8$ is three times greater than $8 \rho^{-1}$ and thus the signal is very likely to be detected. The error is about $1.1^{\circ}$ for $\theta=90^{\circ}$ and is $2.2^{\circ}$ for $\theta=30^{\circ}$.

Now suppose the array is bent but is processed as if it were linear. Then the true position of the $k$ th sensor is generally different from the assumed position on the nominal axis. Equation 2 becomes

$$
S\left(\kappa, \omega_{0}\right)=K^{-1}\left|T^{-1} \sum_{t=1}^{T} \sum_{k=1}^{K} \mathrm{p}\left(t, x_{k}+d_{k}\right) \exp \left[i\left(\kappa x_{k}-\omega_{0} t\right)\right]\right|^{2},
$$

where $d_{k}$ is the displacement due to the projection of the array on the nominal axis. For $\kappa=\kappa_{0}$, the frequency wavenumber spectrum can be written

$$
\begin{aligned}
S\left(\kappa_{0}, \omega_{0}\right)= & \mid A \exp (i \theta) K^{-1 / 2} \sum_{k=1}^{K} \exp \left(-i \kappa_{0} d_{k}\right) \\
& +\left.K^{-1 / 2} \sum_{k=1}^{K} N_{k} \exp \left(i \kappa_{0} x_{k}\right)\right|^{2},
\end{aligned}
$$

where the real and imaginary parts of $N_{k}$ are uncorrelated Gaussian $N\left(0, \sigma^{2} / 2 T\right)$ random variables (for details see Hinich and Shaman $^{1}$ ).

If the array is truly linear, each $d_{k}=0$, and thus $K^{-1 / 2} \sum_{k=1}^{K} \exp \left(i \kappa_{0} d_{k}\right)=K^{1 / 2}$. In order to show the degradation due to bending, consider an example where the odd $d_{k}$ are zero but the even ones are equal to $\lambda / 2$, i. e., $\kappa_{0} d_{k / 2}=\pi$. Thus for even $K$,

$$
K^{-1 / 2} \sum_{k=1}^{K} \exp \left(-i \kappa_{0} d_{k}\right)=-\frac{1}{2} K^{1 / 2}
$$

which means that the effective sensor redundancy is $K / 4$ in the inequality, Eq. 4. Of course, such a pattern of $d_{k}$ 's is unrealistic, but the example indicates the type of effect which is observed in the artificial data results presented later. The $\exp \left(-i \kappa d_{k}\right)$ terms in the frequencywavenumber spectrum equation cause the wave's energy peak to shift away from $\kappa=\kappa_{0}$.

It is possible to reduce the distortion and degradation due to bending by processing the array as a set of smaller aperture subarrays. In many applications, the array aperture is so large and the sensor redundancy is sufficiently great to produce very narrow beams with high array-processing gain, provided the array is linear, the signal is coherent across the array, and the noise is incoherent. When the array is bending, the idea is to trade off aperture for a reduction in the bending bias in order to obtain a bearing which is not wildly fluctuating over time. A pencil thin beam is not essential in many operational situations. The statistical technique for bearing estimation using subarrays is presented in Sec. I.

\section{SUBARRAY PROCESSING}

Suppose that the array is grouped into $J$ subarrays of adjacent hydrophones. Let $\hat{\theta}_{j}$ denote the bearing estimate obtained from $j$ th subarray. Since the nonlinear distortion is less for a section of the array, assume that there is enough structural rigidity in the subarrays so that the maximum likelihood procedure can be used to obtain $\hat{\theta}_{j}$, and that the subarray has sufficient sensor redundancy (or $T$ is sufficiently large) so that the error in $\hat{\theta}_{j}$ due to noise is not large. Let $b_{j}$ denote the inclination of the $j$ th subarray with respect to the axis during the $T \delta$-sec observation period. Then

$$
\hat{\theta}_{j}=\theta+b_{j}+\epsilon_{j},
$$

where $\epsilon_{j}$ is the noise component in the bearing estimate. In order to obtain the theoretical error bounds for the robust procedure, let us model the bending by assuming that $\sum_{j=1}^{J} b_{j} \approx 0$. Further, assume that $\epsilon_{1}, \ldots, \epsilon_{\delta}$ are independent identically distributed random errors whose variance is given by Eq. 3. This assumption is true if the noise is incoherent, but even if there is some spatial coherence in the noise, the statistical model should yield useful approximations for the empirical properties of the simple estimators we now propose.

Given the subarray estimates compute the mean

$$
\bar{\theta}=J^{-1} \sum_{j=1}^{J} \hat{\theta}_{j} \text {. }
$$

Since the errors $\epsilon_{j}$ are uncorrelated, the variance of $\bar{\theta}$ is simply $J^{-1} \sigma_{\epsilon}^{2}$, where $\sigma_{\epsilon}^{2}$ is the variance of the $\epsilon_{j}$. Assuming that $T$ is sufficiently large relative to $A / \sigma$ so that the approximation, Eq. 3, holds for $\sigma_{\epsilon}^{2}$, then

$$
\sigma^{2}(\bar{\theta})=J^{2} \sigma^{2}(\hat{\theta})
$$

if all the subarrays have $K / J$ sensors. Thus the sub-

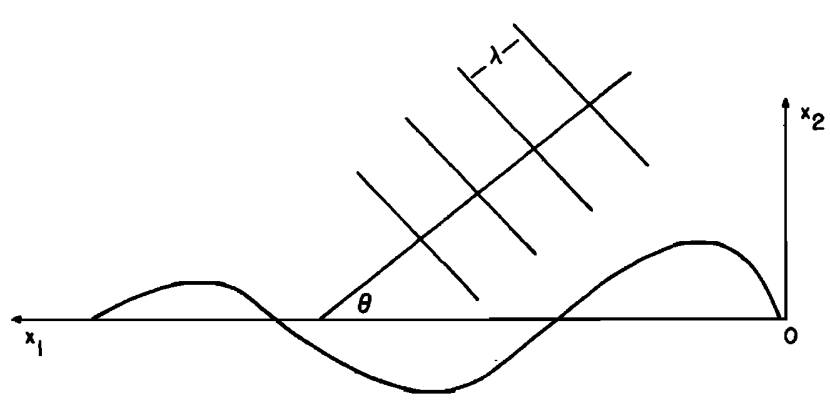

FIG. 2. Incident plane wave detected by array. 
array processing is inferior to the standard processing if the array is truly linear. However, for the sinusoidal patterns which were used in the artificial data study which is discussed in Sec. II., the mean $\vec{\theta}$ outperformed the whole array estimator $\hat{\theta}$.

Since we have $J$ independent but noisy estimates of the true $\theta$, we can extract some information about the magnitude of the array nonlinearity by computing some other statistics using the $\hat{\theta}_{j}$, and we can protest ourselves against some highly spurious subarray bearings.

In contrast to the mean, the median of the $\hat{\theta}_{j}$ 's is fairly insensitive to large errors in the bearings obtained from some subarrays. ${ }^{5}$ The median is superior to the mean when some of the subarray estimates are poor due to local ambient noise or hydrophone failure. Nonetheless, the mean was generally better than the median in our artificial data runs. However, we used a noise model which was consistent with stationary and incoherent Gaussian ambient noise. In a real application, the median is worth looking at. A significant difference between the mean and the median could be due to a second source which is being detected by one part of the array, or it would be due to a malfunction of part of the array.

Another statistic which is useful to look at is the normalized range of variation of the $\hat{\theta}$, bearings. It is computed as follows. Order the residuals $U_{j}=\hat{\theta}_{j}-$ median $\hat{\theta}_{j}$ $(j=1, \ldots, J)$ from the largest to the smallest (including sign). Then compute

$$
\hat{c}=0.855\left(U_{0.72}-U_{0.28}\right),
$$

where $U_{0.72}$ is the residual $U_{j}=\hat{\theta}_{j}-$ median $\hat{\theta}_{j}$, which is the $72 \%$ largest $U_{j}$ and $U_{0.28}$ is the $28 \%$ largest. ${ }^{6}$ For example, if $J=100, U_{0.72}$ is the 72nd largest $U_{j}$. If there is no integer which divides the ordered residuals exactly as prescribed, use an average of two consecutively ordered $U$, around the $72 \%$ and $28 \%$ break point. For example, if $J=101$, use the average of the 72nd and 73rd largest, and similarly for $U_{0.28}$. If the $\hat{\theta}_{j}$ are normally distributed with variance $\sigma_{a}^{2}$, then $\hat{c}$ is a consistent estimator of $\sigma_{a}$ as $J \rightarrow \infty$, and is less subject to distortion than the usual estimator due to some highly spurious values of the subarray bearings. The artificial data results show that the greater the amplitude of the sinusoidal bending, the larger the $\hat{c}$ scale estimates. This is not surprising since a large amount of bending will cause the $\hat{\theta}_{j}$ estimates to have a large range.

In Sec. II, we present artificial data analysis of the mean, median, and the scale $\hat{c}$ when the $d_{k}$ and thus the $b$, biases are derived from an array which has a sinusoidal geometry.

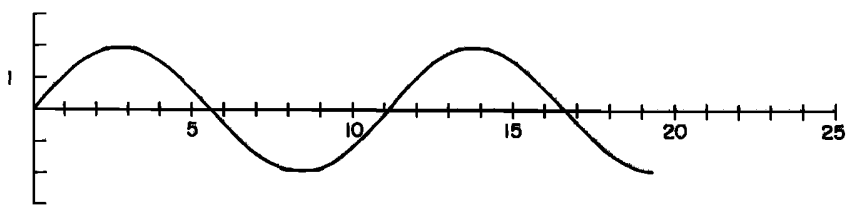

FIG. 3. Sine wave with amplitude equal to 2 .

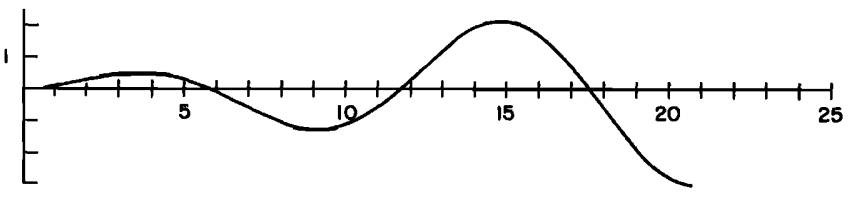

FIG. 4. Damped sine wave.

\section{SIMULATION TESTS OF THE BEARING ESTIMATORS}

Simulation of array processing using digital computer methods is relatively straightforward. Let $\mathrm{x}=\left(x_{1}, x_{2}\right)$ denote a point on the horizontal plane containing the array. The plane-wave signal at $x$ is

$$
s(t, \mathbf{x})=A \exp \left[-i\left(\omega_{0} t-\kappa_{1} x_{1}-\kappa_{2} x_{2}-\phi\right)\right],
$$

where $\kappa_{1}=\left(\omega_{0} / v\right) \cos \theta$ and $\kappa_{2}=\left(\omega_{0} / v\right) \sin \theta$. Since the processor computes the real and imaginary parts of the Fourier transform of $s(t, x)$ and the bearing estimate is the same for all values of the unknown phase $\phi$, the simulations were made with $\phi=0$ but the processing was done as if $\phi$ was unknown. Phase velocity $v$ is assumed to be one, and frequency $\omega_{0}=2 \pi$.

The signal-to-noise ratio in the simulations was varied by fixing the amplitude $A=1$ and multiplying the real and imaginary components of $n$ by a scaling factor $\sigma / 2$. We used $\sigma=1,0.5$, and 0.1 .

The signal is then

$$
s(t, \mathrm{x})=\exp (-i 2 \pi t) \exp \left[i\left(\kappa_{1} x_{1}+\kappa_{2} x_{2}\right)\right] .
$$

The signal is considered to have been filtered in a narrow band about $\omega_{0}$, and thus the $t$ index is dropped for the remainder of this discussion. The components $\left(\kappa_{1}\right.$, $\kappa_{2}$ ) of the wavenumber $\kappa$ are now

$$
\kappa_{1}=2 \pi \cos \theta, \quad \kappa_{2}=2 \pi \sin \theta,
$$

where $\theta=30^{\circ}, 60^{\circ}$, and $90^{\circ}$ were used. Given the frequency $\omega_{0}=2 \pi$, the sensor separation $d$ is set at $\frac{1}{2}$ in order to avoid aliasing problems, i.e.,

$$
d \leqslant \frac{1}{2} \lambda=\frac{1}{2}\left(2 \pi v / \omega_{0}\right)=\frac{1}{2} \text {. }
$$

The noise component of the signal is complex and Gaussian with

$$
\begin{array}{rlrl}
E\left[n\left(x_{j}\right) \bar{n}\left(x_{k}\right)\right] & =\sigma^{2}, & & \text { for } j=k, \\
& =0, & \text { for } j \neq k,
\end{array}
$$

where $n\left(x_{j}\right)$ and $n\left(x_{k}\right)$ represent the noise components received at the $j$ th and $k$ th sensors, respectively.

The model as simulated thus specifies the signal received at sensor $k$ as

$$
p\left(\mathrm{x}_{k}\right)=\exp \left[2 \pi i\left(x_{1 k} \cos \theta+x_{2 k} \sin \theta\right)\right]+n\left(\mathrm{x}_{k}\right),
$$

where $x_{1 k}$ and $x_{2 k}$ specify the location of the $k$ th sensor with respect to the coordinate system moving with the array (refer back to Fig. 2).

Two types of sinusoidal array patterns were used in our simulations: a sine wave of $3 \frac{1}{2}$ half-cycle length and a damped sine wave of $3 \frac{1}{2}$ half-cycles (see Figs. 3 and 4). In all cases, the sensor-to-sensor segments were 


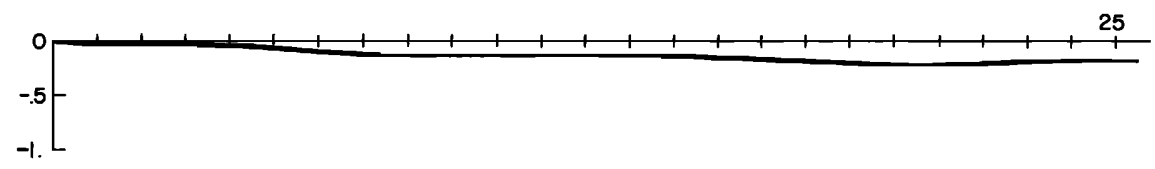

FIG. 5. Random bent array.

made linear with a between sensor separation of $\frac{1}{2}$, and consequently the patterns are only approximately sinusoidal. Fifty sensors were used.

In addition, we tried a random bending pattern which was constructed by fixing an endpoint and setting each successive sensor position $\frac{1}{2}$ unit along a ray from the previous sensor. The angle of this ray with respect to the axis was drawn from a uniform density on the interval $\left(-5^{\circ}, 5^{\circ}\right)$, independently of the other angles. Thus the maximum angular deflection at each node is $\pm 5^{\circ}$. The pattern which resulted from this process and which was used in the simulation is shown in Fig. 5. It can be seen that most sensors were on one side of the axis and that the maximum displacement of the array was 0.25 , which is one-half of the between-sensor spacing.

The undamped sine wave pattern was used with four different amplitudes, namely, $\frac{1}{2}, 1,2$, and 3 . Even in the least bending model (when the maximum deviation from the axis was equal to the sensor spacing of $\frac{1}{2}$ ) the average bearing computed from the 50-element array was significantly different from the true bearing. The terminal amplitude of the damped sine wave were $\frac{1}{2}, 1$, 2, and 3. Note that we biased the pattern against our subarray processing method by having more sensors on one side of the nominal axis than the other. We also tried sinusoidal patterns which had two full cycles and thus had a symmetric distribution of sensors about the nominal axis. The results using these patterns were similar to the ones reported.

Independent random $N(0,1)$ deviates were generated in pairs using the Box and Muller transformation, ${ }^{7}$

$$
N_{1}=\left(-2 \ln U_{1}\right)^{1 / 2} \cos 2 \pi U_{2}, \quad N_{2}=\left(-2 \ln U_{1}\right)^{1 / 2} \sin 2 \pi U_{2},
$$

where $U_{1}$ and $U_{2}$ are independent uniform $(0,1)$ variates. These uniform variates, in turn, were generated using the power residue method. ${ }^{8}$ The $N(0,1)$ deviates were then scaled to display the desired variance.

The subarray methods used 12 nonoverlapping foursensor arrays plus two end arrays which overlapped in order to use all the $\mathbf{5 0}$ sensors, and six nonoverlapping eight-sensor arrays plus two overlapping end arrays. We also tried successively overlapped subarray patterns, but the results were essentially the same. Subarray bearing estimates are made by augmenting the subarray's sensors' outputs with zeroes and taking the fast Fourier transform of the augmented subarray of complex sensor outputs. After transformation, the subarray bearing estimate is selected corresponding to the wavenumber $\hat{\kappa}$ which maximizes the amplitude of the subarray power spectrum.

The FFT routine used required an odd number of input points. In the tradeoff between speed and accuracy, it was decided to apply the FFT to records of 101 points length obtained by augmenting the four - or eight-subarray points with zeroes. The maximum quantization error thus introduced in $\hat{\kappa}$ is $\pm 2 \pi / 101 d$, which translated into an error in $\hat{\theta}$ of about $0.6^{\circ}$. This error is larger than the $\sigma(\hat{\theta})$ due to the noise.

Two methods were used in determining the final bearing estimate. The first was the median of the subarray estimates; and the second was the mean of the subarray estimates. In addition, the scale estimate $\hat{c}$ was calculated as previously discussed.

This process was repeated 100 times for a given true bearing angle and noise variance with the generation of new noise components for the signals received at each sensor. Averages of the median-derived and mean-derived bearing estimates were calculated as well as for the scale estimate. In addition, rms errors about the true bearing angle were calculated for each bearing estimate method. Our results are presented in Tables I and II.

The results of the simulations were somewhat equivocal in determining the estimator of choice vis-a-vis the median- or mean-derived estimators. It is clear, however, that under a broad variety of array patterns and signal-to-noise environments, the subarray processing is superior to the full array technique.

The effect of deflection bias on the full-array estimate is generally quite significant, even under very favorable circumstances. Consider the case of the full-array estimator using the undamped wave with an amplitude of $\frac{1}{2}$, $\sigma=0.5$, and $\theta=90^{\circ}$. In this case, the variance of the 100 estimates is 20.7 and the bias is 6.7. The estimated standard error of the mean is 0.455 and, hence the bias is 14.7 times the standard error of the mean. Thus even under relatively favorable conditions the mean estimated bearing angle is very significantly different from the true bearing angle. It should also be noted that as the signal-to-noise ratio increases, the variance tends to zero-while the bias tends to increase with the effect that the full-array mean estimates diverge from the true bearing with increasing significance.

With respect to the undamped sine pattern, there is a marked superiority in terms of rms error in the medianderived estimates for true bearing angles of $60^{\circ}$ and $90^{\circ}$. At the $30^{\circ}$ bearing angle, we observe results which are somewhat mixed though they tend to favor the mean-derived estimator. For most applications, the four-element subarray estimator seems to be superior at larger bearing angles, and the eight-element subarray estimator appears better at the smaller bearing angle.

The results show a shift in the estimator of choice from the eight-element to the four-element subarray processing as the true bearing angle increases. Moreover, the mean-derived estimator seems most accurate in most applications. 
TABLE I. Results for undamped sinusoidal bending.

\begin{tabular}{|c|c|c|c|c|c|c|c|c|c|c|c|c|c|c|c|c|c|}
\hline \multirow[b]{2}{*}{$A$} & \multirow[b]{2}{*}{$\sigma$} & \multirow[b]{2}{*}{$K$} & \multicolumn{5}{|c|}{$30^{\circ}$} & \multicolumn{5}{|c|}{$60^{\circ}$} & \multicolumn{5}{|c|}{$90^{\circ}$} \\
\hline & & & med & rms & $\hat{c}$ & mean & rms & med & rms & $\hat{\boldsymbol{c}}$ & mean & $\mathrm{rms}$ & med & $\mathrm{rms}$ & $\hat{c}$ & mean & rms \\
\hline \multirow[t]{3}{*}{$\frac{1}{2}$} & 1.0 & 50 & 32.8 & 7.4 & N/A & 32.8 & 7.4 & 63.6 & 8.1 & $\mathrm{~N} / \mathrm{A}$ & 63.6 & 8.1 & 94.7 & 8.5 & N/A & 94.7 & 8.5 \\
\hline & & 8 & 33.0 & 4.7 & 9.5 & 35.9 & 10.8 & 62.6 & 4.3 & 9.6 & 62.7 & 5.6 & 92.4 & 3.8 & 9.4 & 91.4 & 4.4 \\
\hline & & 4 & 40.5 & 14.2 & 17.9 & 56.3 & 29.0 & 63.6 & 5.4 & 11.8 & 67.4 & 9.8 & 91.0 & 3.8 & 11.6 & 89.4 & 5.4 \\
\hline \multirow[t]{3}{*}{$\frac{1}{2}$} & 0.5 & 50 & 33.2 & 7.3 & N/A & 33.2 & 7.3 & 66.3 & 8.6 & N/A & 66.3 & 8.6 & 96.7 & 8.1 & N/A & 96.7 & 8.1 \\
\hline & & 8 & 31.9 & 2.4 & 9.5 & 29.9 & 1.2 & 62.4 & 2.6 & 9.2 & 60.8 & 0.9 & 92.8 & 2.9 & 9.2 & 91.2 & 1.3 \\
\hline & & 4 & 32.7 & 4.0 & 9.2 & 36.4 & 9.5 & 61.2 & 2.3 & 9.4 & 60.2 & 1.2 & 91.9 & 2.5 & 9.9 & 90.6 & 1.2 \\
\hline \multirow[t]{3}{*}{$\frac{1}{2}$} & 0.1 & 50 & 36.7 & 6.9 & N/A & 36.7 & 6.9 & 68.9 & 8.9 & N/A & 68.9 & 8.9 & 98.0 & 8.0 & N/A & 98.0 & 8.0 \\
\hline & & 8 & 32.0 & 2.0 & 9.6 & 30.0 & 0.2 & 62.6 & 2.6 & 9.1 & 60.8 & 0.8 & 92.8 & 2.8 & 9.2 & 91.1 & 1.1 \\
\hline & & 4 & 30.7 & 1.1 & 10.8 & 29.5 & 0.7 & 61.2 & 1.3 & 10.7 & 60.3 & 0.4 & 91.5 & 1.6 & 10.7 & 90.6 & 0.6 \\
\hline \multirow[t]{3}{*}{1} & 1.0 & 50 & 32.7 & 21.7 & N/A & 32.7 & 21.7 & 64.8 & 17.4 & N/A & 64.8 & 17.4 & 100.2 & 19.1 & N/A & 100.2 & 19.1 \\
\hline & & 8 & 41.6 & 13.1 & 18.8 & 48.7 & 24.0 & 66.1 & 9.2 & 19.3 & 64.5 & 8.0 & 92.1 & 8.8 & 20.2 & 90.8 & 5.6 \\
\hline & & 4 & 50.2 & 24.5 & 24.9 & 64.5 & 37.5 & 66.2 & 9.5 & 19.1 & 66.9 & 9.6 & 91.1 & 8.0 & 20.2 & 89.5 & 5.6 \\
\hline \multirow[t]{3}{*}{1} & 0.5 & 50 & 31.2 & 17.6 & $\mathrm{~N} / \mathrm{A}$ & 31.2 & 17.6 & 73.5 & 18.9 & $\mathrm{~N} / \mathrm{A}$ & 73.5 & 18.9 & 107.3 & 20.2 & $\mathrm{~N} / \mathrm{A}$ & 107.3 & 20.2 \\
\hline & & 8 & 38.7 & 9.6 & 18.2 & 40.3 & 15.4 & 66.0 & 6.3 & 19.1 & 62.1 & 2.3 & 95.5 & 6.3 & 19.3 & 92.1 & 2.4 \\
\hline & & 4 & 42.5 & 13.6 & 18.2 & 54.2 & 28.0 & 63.5 & 4.1 & 20.5 & 60.9 & 1.4 & 93.3 & 3.9 & 21.3 & 91.4 & 1.8 \\
\hline \multirow[t]{3}{*}{1} & 0.1 & 50 & 31.5 & 17.8 & N/A & 31.5 & 17.8 & 79.6 & 19.6 & N/A & 79.6 & 19.6 & 111.1 & 21.1 & N/A & 111.1 & 21.1 \\
\hline & & 8 & 35.9 & 6.0 & 19.6 & 31.3 & 2.6 & 65.8 & 5.8 & 19.1 & 62.0 & 2.0 & 95.7 & 5.8 & 19.1 & 92.2 & 2.2 \\
\hline & & 4 & 37.6 & 9.0 & 19.8 & 39.4 & 14.3 & 62.8 & 2.8 & 21.9 & 60.9 & 1.0 & 93.0 & 3.0 & 21.9 & 91.3 & 1.3 \\
\hline \multirow[t]{3}{*}{2} & 1.0 & 50 & 32.5 & 29.5 & N/A & 32.5 & 29.5 & 61.7 & 42.7 & $\mathrm{~N} / \mathrm{A}$ & 61.7 & 42.7 & 113.2 & 41.9 & N/A & 113.2 & 41.9 \\
\hline & & 8 & 53.8 & 28.9 & 29.8 & 56.0 & 30.5 & 80.5 & 27.5 & 39.0 & 74.4 & 20.0 & 97.7 & 20.3 & 41.2 & 93.5 & 10.0 \\
\hline & & 4 & 65.8 & 37.9 & 30.8 & 71.3 & 43.8 & 86.7 & 29.9 & 34.3 & 82.9 & 25.9 & 93.0 & 16.7 & 41.1 & 90.6 & 7.2 \\
\hline \multirow[t]{3}{*}{2} & 0.5 & 50 & 15.2 & 19.3 & $\mathrm{~N} / \mathrm{A}$ & 15.2 & 19.3 & 59.9 & 41.5 & N/A & 59.9 & 41.5 & 130.2 & 43.8 & N/A & 130.2 & 43.8 \\
\hline & & 8 & 48.2 & 22.0 & 31.9 & 47.4 & 19.6 & 75.7 & 21.5 & 42.5 & 66.5 & 9.2 & 106.5 & 21.1 & 45.5 & 95.1 & 6.4 \\
\hline & & 4 & 55.9 & 27.8 & 29.2 & 57.1 & 30.2 & 83.5 & 26.3 & 38.3 & 75.3 & 19.0 & 98.5 & 9.3 & 47.5 & 93.1 & 3.5 \\
\hline \multirow[t]{3}{*}{2} & 0.1 & 50 & 11.5 & 18.5 & $\mathrm{~N} / \mathrm{A}$ & 11.5 & 18.5 & 52.8 & 36.9 & N/A & 52.8 & 36.9 & 136.0 & 16.0 & N/A & 136.0 & 46.0 \\
\hline & & 8 & 47.3 & 19.0 & 31.1 & 44.2 & 14.6 & 80.7 & 22.5 & 43.3 & 66.8 & 7.2 & 116.9 & 26.9 & 47.0 & 96.7 & 6.7 \\
\hline & & 4 & 39.2 & 9.2 & 30.3 & 41.9 & 11.9 & 68.4 & 8.6 & 48.5 & 62.6 & 2.9 & 97.8 & 7.8 & 48.4 & 93.3 & 3.3 \\
\hline \multirow[t]{3}{*}{3} & 1.0 & 50 & 63.5 & 45.5 & N/A & 63.5 & 45.5 & 111.0 & 72.2 & N/A & 111.0 & 72.2 & 163.3 & 84.3 & $\mathrm{~N} / \mathrm{A}$ & 163.3 & 84.3 \\
\hline & & 8 & 73.1 & 47.2 & 32.9 & 70.5 & 41.6 & 113.0 & 57.7 & 52.1 & 95.4 & 39.1 & 110.8 & 43.4 & 70.3 & 97.2 & 18.0 \\
\hline & & 4 & 74.3 & 47.2 & 31.8 & 71.8 & 42.5 & 112.5 & 55.5 & 41.5 & 100.5 & 42.6 & 94.5 & 32.6 & 64.4 & 91.2 & 13.4 \\
\hline \multirow[t]{3}{*}{3} & 0.5 & 50 & 59.7 & 42.1 & N/A & 59.7 & 42.1 & 114.2 & 67.5 & N/A & 114.2 & 67.5 & 178.3 & 88.5 & N/A & 178.3 & 88.5 \\
\hline & & 8 & 74.9 & 47.5 & 35.4 & 69.2 & 39.7 & 121.6 & 62.9 & 59.7 & 94.1 & 36.9 & 140.2 & 57.1 & 81.8 & 104.5 & 17.0 \\
\hline & & 4 & 65.1 & 36.0 & 34.7 & 66.2 & 36.3 & 121.3 & 62.1 & 50.4 & 99.0 & 41.3 & 104.1 & 38.6 & 78.4 & 92.8 & 9.6 \\
\hline \multirow[t]{3}{*}{3} & 0.1 & 50 & 50.9 & 33.4 & N/A & 50.9 & 33.4 & 128.5 & 68.5 & N/A & 128.5 & 68.5 & 179.9 & 89.9 & N/A & 179.9 & 89.9 \\
\hline & & 8 & 70.9 & 42.0 & 34.9 & 67.9 & 38.0 & 124.2 & 65.1 & 65.7 & 92.6 & 34.6 & 156.6 & 66.6 & 81.5 & 109.9 & 19.9 \\
\hline & & 4 & 61.1 & 31.1 & 33.8 & 65.3 & 35.3 & 101.7 & 45.9 & 70.4 & 77.3 & 18.5 & 90.4 & 0.9 & 81.4 & 89.4 & 0.8 \\
\hline
\end{tabular}

It is not surprising that the four-element subarray gives poorer results than the eight-element configuration when $\theta=30^{\circ}$. The greater sidelobes of the smaller aperture configuration results in a sidelobe induced variance for targets whose position is near endfire which more than outweighs the greater reduction of the bending deflection due to averaging more smaller subarrays. For the parameters selected in this experiment, the trade-off between subarray aperture and deflection bias is in favor of the eight element length for $\theta=30^{\circ}$.

For the broadside case $\left(\theta=90^{\circ}\right)$, however, the situation is reversed. The four element array average is better except for the $\sigma=1$ case in the $A=\frac{1}{2}$ patterns.

Examining the results for the undamped and damped sine patterns, one point is especially clear: Among all estimators at all signal-to-noise ratios and at all bearing angles, the full array estimator is almost universally the poorest estimator of true bearing angle. To be sure, there are some instances in Tables $I$ and $I I$ in which the full-array estimate shows the smallest deflection, but in only isolated and evidently unsystematic instances does the full-array estimator demonstrate lowest rms error. As a case in point consider the undamped sine wave of amplitude 2 and unit error with a true bearing angle of $30^{\circ}$. The average of 100 full-array bearing estimates is $32.5^{\circ}$. However the rms error is $29.5^{\circ}$, which yields a standard deviation of $29.4^{\circ}$. Thus the standard error of the mean is $2.94^{\circ}$, making the deflection statistically insignificant.

The choice of recommended estimator, then, is between the eight- and four-element subarray estimators, and between the median- and mean-derived estimators.

The theoretical sensitivity of the mean to large deviations would seem to argue in favor of the median-derived estimator. However, the results of the simulations, particularly using the damped sine wave pattern, do not unequivocally affirm this sensitivity of the mean.

The choice among subarray size and processing combinations is one which would probably be made in a particular application according to the extent of bending expected and to signal-to-noise ratios anticipated. 
TABLE II. Results for damped sinusoidal bending.

\begin{tabular}{|c|c|c|c|c|c|c|c|c|c|c|c|c|c|c|c|c|c|}
\hline \multirow[b]{2}{*}{$A$} & \multirow[b]{2}{*}{$\sigma$} & \multirow[b]{2}{*}{$K$} & \multicolumn{5}{|c|}{$30^{\circ}$} & \multicolumn{5}{|c|}{$60^{\circ}$} & \multicolumn{5}{|c|}{$90^{\circ}$} \\
\hline & & & med & $\mathbf{r m s}$ & $\hat{\boldsymbol{c}}$ & mean & $\mathrm{rms}$ & med & $\mathrm{rms}$ & $\hat{\boldsymbol{c}}$ & mean & rms & med & $\mathrm{rms}$ & $\hat{\boldsymbol{c}}$ & mean & $\mathrm{rms}$ \\
\hline \multirow[t]{3}{*}{$\frac{1}{2}$} & 1.0 & 50 & 29.7 & 1.2 & $\mathrm{~N} / \mathrm{A}$ & 29.7 & 1.2 & 59.6 & 3.4 & N/A & 59.6 & 3.4 & 90.3 & 3.8 & N/A & 90.3 & 3.8 \\
\hline & & 8 & 31.4 & 2.9 & 5.1 & 34.5 & 8.3 & 61.4 & 2.4 & 4.3 & 62.8 & 5.2 & 91.6 & 2.2 & 4.6 & 91.5 & 4.6 \\
\hline & & 4 & 36.8 & 9.4 & 15.0 & 50.4 & 23.3 & 62.5 & 4.1 & 7.9 & 67.3 & 9.3 & 91.3 & 3.3 & 7.8 & 90.8 & 5.5 \\
\hline \multirow[t]{3}{*}{$\frac{1}{2}$} & 0.5 & 50 & 29.7 & 1.2 & $\mathrm{~N} / \mathrm{A}$ & 29.7 & 1.2 & 59.3 & 2.0 & N/A & 59.3 & 2.0 & 89.2 & 3.4 & $\mathrm{~N} / \mathrm{A}$ & 89.2 & 3.4 \\
\hline & & 8 & 30.5 & 1.4 & 3.4 & 30.8 & 1.1 & 61.3 & 1.5 & 2.8 & 61.5 & 1.6 & 91.6 & 1.7 & 2.8 & 91.8 & 1.8 \\
\hline & & 4 & 30.8 & 2.2 & 5.7 & 31.0 & 4.0 & 60.9 & 1.6 & 4.2 & 61.0 & 1.4 & 91.3 & 1.7 & 4.2 & 91.3 & 1.5 \\
\hline \multirow[t]{3}{*}{$\frac{1}{2}$} & 0.1 & 50 & 30.1 & 1.0 & $\mathrm{~N} / \mathrm{A}$ & 30.1 & 1.0 & 59.8 & 0.5 & N/A & 59.8 & 0.5 & 87.8 & 3.0 & N/A & 87.8 & 3.0 \\
\hline & & 8 & 30.9 & 1.0 & 2.4 & 30.9 & 1.0 & 61.8 & 1.8 & 2.4 & 61.6 & 1.6 & 91.7 & 1.7 & 2.2 & 91.8 & 1.8 \\
\hline & & 4 & 29.7 & 0.9 & 3.7 & 30.4 & 0.5 & 60.1 & 0.5 & 3.4 & 61.0 & 1.0 & 90.4 & 0.6 & 3.4 & 91.3 & 1.3 \\
\hline \multirow[t]{3}{*}{1} & 1.0 & 50 & 29.9 & 14.7 & N/A & 29.9 & 14.7 & 61.7 & 5.6 & N/A & 61.7 & 5.6 & 92.4 & 6.3 & N/A & 92.4 & 6.3 \\
\hline & & 8 & 33.9 & 5.7 & 9.1 & 38.4 & 12.8 & 63.5 & 4.7 & 7.6 & 64.8 & 6.7 & 93.0 & 4.2 & 8.3 & 93.4 & 6.3 \\
\hline & & 4 & 40.3 & 12.0 & 16.9 & 53.6 & 25.9 & 64.6 & 6.5 & 10.9 & 68.3 & 10.2 & 91.9 & 4.7 & 11.0 & 91.4 & 6.0 \\
\hline \multirow[t]{3}{*}{1} & 0.5 & 50 & 28.4 & 1.7 & N/A & 28.4 & 1.7 & 62.3 & 2.8 & N/A & 62.3 & 2.8 & 92.1 & 2.4 & N/A & 92.1 & 2.4 \\
\hline & & 8 & 32.7 & 3.0 & 6.1 & 32.6 & 2.8 & 63.2 & 3.4 & 5.4 & 63.3 & 3.4 & 93.7 & 3.8 & 5.4 & 93.7 & 3.7 \\
\hline & & 4 & 33.2 & 4.1 & 8.3 & 37.8 & 10.6 & 61.7 & 2.4 & 7.8 & 62.3 & 2.6 & 91.8 & 2.4 & 7.7 & 92.6 & 2.9 \\
\hline \multirow[t]{3}{*}{1} & 0.1 & 50 & 28.4 & 1.6 & N/A & 28.4 & 1.6 & 62.6 & 2.6 & N/A & 62.6 & 2.6 & 92.3 & 2.3 & $\mathbf{N} / \mathbf{A}$ & 92.3 & 2.3 \\
\hline & & 8 & 33.1 & 3.1 & 5.5 & 32.6 & 2.6 & 63.7 & 3.7 & 4.9 & 63.4 & 3.4 & 93.9 & 4.0 & 5.0 & 93.7 & 3.7 \\
\hline & & 4 & 29.9 & 0.8 & 7.0 & 31.6 & 1.7 & 60.4 & 0.7 & 6.7 & 62.4 & 2.4 & 90.6 & 0.7 & 6.8 & 92.7 & 2.7 \\
\hline \multirow[t]{3}{*}{2} & 1.0 & 50 & 35.8 & 37.7 & $\mathbf{N} / \mathbf{A}$ & 35.8 & 37.7 & 64.2 & 17.5 & $\mathbf{N} / \mathbf{A}$ & 64.2 & 17.5 & 94.5 & 17.2 & N/A & 94.5 & 17.2 \\
\hline & & 8 & 42.8 & 15.4 & 19.5 & 51.9 & 24.7 & 66.3 & 9.6 & 17.3 & 68.5 & 10.6 & 94.7 & 7.6 & 17.6 & 96.2 & 8.0 \\
\hline & & 4 & 50.7 & 23.0 & 24.8 & 64.5 & 36.6 & 67.6 & 10.3 & 18.1 & 70.3 & 12.2 & 93.2 & 6.8 & 19.0 & 93.7 & 6.7 \\
\hline \multirow[t]{3}{*}{2} & 0.5 & 50 & 26.4 & 22.6 & $\mathbf{N} / \mathbf{A}$ & 26.4 & 22.6 & 65.7 & 11.3 & N/A & 65.7 & 11.3 & 93.0 & 9.3 & $\mathrm{~N} / \mathrm{A}$ & 93.0 & 9.3 \\
\hline & & 8 & 40.3 & 11.5 & 17.5 & 47.9 & 20.9 & 65.9 & 6.4 & 14.7 & 67.4 & 7.5 & 95.9 & 6.6 & 14.7 & 97.5 & 7.7 \\
\hline & & 4 & 39.8 & 11.0 & 17.1. & 49.0 & 21.1 & 63.7 & 4.5 & 15.1 & 65.2 & 5.5 & 93.9 & 4.4 & 15.0 & 95.9 & 6.0 \\
\hline \multirow[t]{3}{*}{2} & 0.1 & 50 & 19.9 & 10.1 & N/A & 19.9 & 10.1 & 71.3 & 11.3 & N/A & 71.3 & 11.3 & 90.1 & 1.3 & N/A & 90.1 & 1.3 \\
\hline & & 8 & 39.9 & 10.7 & 16.7 & 45.9 & 19.3 & 66.8 & 6.8 & 13.4 & 67.3 & 7.3 & 96.9 & 6.9 & 13.2 & 97.4 & 7.4 \\
\hline & & 4 & 37.6 & 8.5 & 16.7 & 45.1 & 17.7 & 63.5 & 3.5 & 14.2 & 65.4 & 5.4 & 93.6 & 3.7 & 14.1 & 95.8 & 5.8 \\
\hline \multirow[t]{3}{*}{3} & 1.0 & 50 & 27.0 & 27.4 & $\mathbf{N} / \mathbf{A}$ & 27.0 & 27.4 & 58.1 & 28.8 & $\mathbf{N} / \mathbf{A}$ & 58.1 & 28.8 & 104.0 & 32.7 & $\mathbf{N} / \mathbf{A}$ & 104.0 & 32.7 \\
\hline & & 8 & 50.7 & 24.8 & 29.6 & 54.4 & 27.9 & 71.2 & 16.5 & 28.5 & 72.4 & 14.8 & 98.4 & 13.8 & 29.2 & 98.8 & 11.6 \\
\hline & & 4 & 60.5 & 32.2 & 29.1 & 69.2 & 41.2 & 76.1 & 19.6 & 25.9 & 76.7 & 19.0 & 96.9 & 10.9 & 27.7 & 95.4 & 8.6 \\
\hline \multirow[t]{3}{*}{3} & 0.5 & 50 & 13.1 & 19.6 & N/A & 13.1 & 19.6 & 56.5 & 21.7 & N/A & 56.5 & 21.7 & 109.5 & 30.7 & $\mathrm{~N} / \mathrm{A}$ & 109.5 & 30.7 \\
\hline & & 8 & 41.1 & 13.7 & 29.7 & 45.6 & 17.3 & 68.3 & 8.6 & 31.3 & 70.5 & 10.7 & 99.5 & 9.9 & 33.1 & 100.5 & 10.7 \\
\hline & & 4 & 51.3 & 22.2 & 24.5 & 57.1 & 29.4 & 73.7 & 14.7 & 23.6 & 71.3 & 12.5 & 101.7 & 12.0 & 23.5 & 98.7 & 8.8 \\
\hline \multirow[t]{3}{*}{3} & 0.1 & 50 & 11.5 & 18.5 & N/A & 11.5 & 18.5 & 51.0 & 10.4 & N/A & 51.0 & 10.4 & 112.3 & 22.3 & N/A & 112.3 & 22.3 \\
\hline & & 8 & 37.1 & 7.1 & 28.9 & 42.5 & 12.5 & 67.6 & 7.6 & 30.6 & 70.8 & 10.8 & 97.9 & 8.0 & 37.1 & 98.9 & 8.9 \\
\hline & & 4 & 46.7 & 17.6 & 23.3 & 47.4 & 19.4 & 71.9 & 11.9 & 22.9 & 68.3 & 8.3 & 102.0 & 12.0 & 22.6 & 98.7 & 8.7 \\
\hline
\end{tabular}

The existence of bending deflection is clear in the tables. In several instances, in fact, the rms error is entirely bias with essentially zero variance as in the eight-element mean estimator using a damped wave with amplitude $1, \sigma=0.1$, and $\theta=30^{\circ}$.

The results for the randomly bent array is given in Table III. We tried more values for $\sigma$. The slight displacement from the nominal axis produced a slight deflection bias for the full array method. The deflection is $1.1^{\circ}$ for $\theta=90^{\circ}, 0.7^{\circ}$ for $\theta=30^{\circ}$, and is at most $0.6^{\circ}$ for $\theta=60^{\circ}$. Part of this small bias is due to interaction with the quantization error induced by using only 101 values for the FFT. However, we did not have enough computer time to add more zeroes in order to reduce the quantization error since each simulation set ran almost thirty minutes on our IBM 360-50.

An alternative to processing adjacent fixed aperture subarrays is to form subarrays by combining two or four sensors from each end of the array, effectively introducing a set of closing aperture subarrays. We at- tempted this alternative method with relatively poor results. Under the most favorable conditions, the closing aperture technique yielded results inferior to fixed aperture processing. Specifically, using the undamped sinusoid with an amplitude of $\frac{1}{2}$ and a bearing angle of $90^{\circ}$ the closing aperture technique yielded rms values for both the median- and mean-derived estimators which were greater than for fixed aperture processing using the same number of subarray elements. The observed rms values for the two techniques showed a tendency to converge as the signal-to-noise ratio increased, but in all instances the errors using the closing aperture procedure exceeded those of the fixed aperture procedure. Thus for our simulations, the closing aperture technique is inferior to the fixed aperture, adjacent subarray-averaging method.

\section{ACKNOWLEDGMENT}

This research was supported by the Office of Naval Research under contract. We thank Carl Bennett of the USNCSL for stimulating our interest in this problem and 
TABLE III. Results for random bending pattern.

\begin{tabular}{|c|c|c|c|c|c|c|c|c|c|c|c|c|c|c|c|c|}
\hline \multirow[b]{2}{*}{$\sigma$} & \multirow[b]{2}{*}{$K$} & \multicolumn{5}{|c|}{$30^{\circ}$} & \multicolumn{5}{|c|}{$60^{\circ}$} & \multicolumn{5}{|c|}{$90^{\circ}$} \\
\hline & & med & $\mathrm{rms}$ & $\hat{\boldsymbol{c}}$ & mean & rms & med & $\mathrm{rms}$ & $\hat{\boldsymbol{c}}$ & mean & $\mathrm{rms}$ & med & $\mathrm{rms}$ & $\hat{\boldsymbol{c}}$ & mean & $\mathrm{rms}$ \\
\hline \multirow{3}{*}{1.0} & 50 & 30.6 & 0.8 & N/A & 30.6 & 0.8 & 60.6 & 0.9 & N/A & 60.6 & 0.9 & 91.1 & 1.1 & N/A & 91.1 & 1.1 \\
\hline & 8 & 30.4 & 1.2 & 3.7 & 33.6 & 5.8 & 60.6 & 0.9 & 2.2 & 62.0 & 3.4 & 90.8 & 1.0 & 2.2 & 90.6 & 3.4 \\
\hline & 4 & 35.9 & 6.9 & 15.1 & 50.9 & 22.3 & 61.9 & 2.4 & 6.9 & 67.3 & 8.2 & 90.7 & 1.6 & 6.1 & 90.1 & 3.2 \\
\hline \multirow{3}{*}{0.75} & 50 & 30.7 & 0.7 & N/A & 30.7 & 0.7 & 60.6 & 0.9 & $\mathbf{N} / \mathbf{A}$ & 60.6 & 0.9 & 91.1 & 1.1 & N/A & 91.1 & 1.1 \\
\hline & 8 & 30.3 & 0.9 & 2.5 & 30.0 & 1.0 & 60.5 & 0.8 & 1.6 & 60.5 & 0.6 & 90.8 & 1.0 & 1.5 & 90.8 & 1.1 \\
\hline & 4 & 31.5 & 2.2 & 7.2 & 38.3 & 10.0 & 60.8 & 1.2 & 4.4 & 63.0 & 4.0 & 90.8 & 1.1 & 3.9 & 90.7 & 2.0 \\
\hline \multirow{3}{*}{0.5} & 50 & 30.7 & 0.7 & N/A & 30.7 & 0.7 & 60.3 & 0.7 & N/A & 60.3 & 0.7 & 91.1 & 1.1 & $\mathrm{~N} / \mathrm{A}$ & 91.1 & 1.1 \\
\hline & 8 & 30.5 & 0.8 & 1.6 & 29.8 & 0.3 & 60.5 & 0.8 & 1.1 & 60.4 & 0.4 & 91.0 & 1.1 & 1.1 & 90.7 & 0.7 \\
\hline & 4 & 30.0 & 1.2 & 4.6 & 30.2 & 1.2 & 60.6 & 0.9 & 2.8 & 60.4 & 0.6 & 90.8 & 1.0 & 2.5 & 90.7 & 0.8 \\
\hline \multirow{3}{*}{0.25} & 50 & 30.7 & 0.7 & N/A & 30.7 & 0.7 & 60.1 & 0.4 & N/A & 60.1 & 0.4 & 91.1 & 1.1 & $\mathrm{~N} / \mathrm{A}$ & 91.1 & 1.1 \\
\hline & 8 & 30.6 & 0.7 & 1.3 & 29.8 & 0.2 & 60.6 & 0.9 & 0.8 & 60.4 & 0.4 & 91.1 & 1.1 & 0.8 & 90.7 & 0.7 \\
\hline & 4 & 30.2 & 0.9 & 2.3 & 29.7 & 0.3 & 60.4 & 0.7 & 1.7 & 60.4 & 0.5 & 91.0 & 1.1 & 1.7 & 90.8 & 0.8 \\
\hline \multirow{3}{*}{0.1} & 50 & 30.7 & 0.7 & N/A & 30.7 & 0.7 & 60.0 & 0.0 & N/A & 60.0 & 0.0 & 91.1 & 1.1 & N/A & 91.1 & 1.1 \\
\hline & 8 & 30.7 & 0.7 & 1.2 & 29.9 & 0.2 & 60.5 & 0.8 & 0.8 & 60.4 & 0.4 & 91.1 & 1.1 & 0.7 & 90.7 & 0.7 \\
\hline & 4 & 30.7 & 0.7 & 1.4 & 29.8 & 0.2 & 60.6 & 0.9 & 1.0 & 60.5 & 0.5 & 91.1 & 1.1 & 1.2 & 90.8 & 0.8 \\
\hline
\end{tabular}

for making valuable suggestions, and the referees for their insistence on precision.

\section{APPENDIX A.}

Consider an equally spaced linear array of $K$ sensors. The spatial noise field is assumed to be constant, independent of $\kappa$. For $\kappa_{0}=\left(\omega_{0} / v\right) \cos \theta$, the frequency-wavenumber spectrum is

$$
S\left(\kappa_{0}, \omega_{0}\right)=K^{-1}\left|K A \exp i \phi+\sum_{k=1}^{K} N_{k} \exp i \kappa x_{k}\right|^{2},
$$

where $\operatorname{Re} N_{k}$ and $\operatorname{Im} N_{k}$ are independent, Gaussian $N\left(0, \sigma^{2}\right)$ $2 T)$ random variables. Define

$$
\begin{aligned}
& Z_{R}(\kappa)=\operatorname{Re}\left(K^{-1 / 2} \sum_{k=1}^{K} N_{k} \exp i \kappa x_{k}\right), \\
& Z_{I}(\kappa)=\operatorname{Im}\left(K^{-1 / 2} \sum_{k=1}^{K} N_{k} \exp i \kappa x_{k}\right),
\end{aligned}
$$

which are independent, Gaussian $N\left(0, \sigma^{2} / 2 T\right)$ variables. If $\kappa \neq \kappa^{\prime}, Z_{R}(\kappa)$, and $Z_{R}\left(\kappa^{\prime}\right)$ are uncorrelated for large $K$, and similarly for $Z_{I}$.

$$
\begin{aligned}
& \text { For } \kappa=\kappa_{0}, \\
& \qquad S\left(\kappa_{0}, \omega_{0}\right)=K A^{2}+2 K^{1 / 2} A\left(Z_{R} \cos \phi+Z_{I} \sin \phi\right)+Z_{R}^{2}+Z_{I}^{2} .
\end{aligned}
$$

The third term $\left(Z_{R}^{2}+Z_{I}^{2}\right)$ in Eq. A2 is $\left(\sigma^{2} / 2 T\right) \chi_{2}^{2}$, where $\chi_{2}^{2}$ is a chi-squared random variable, and is of the order $0(1)$, whereas the first term is $O(K)$ and the second $0\left(K^{1 / 2}\right)$.

The variable $Z=Z_{R} \cos \phi+Z_{I} \sin \phi$ is Gaussian $N\left(0, \sigma^{2} /\right.$ 2T). Thus

$$
K A^{2}+2 K^{1 / 2} A Z>K A^{2}-4 K^{1 / 2} A \sigma(2 T)^{-1 / 2},
$$

with probability 0.97 (the probability of being greater than two standard deviations below the mean for a Gaussian distribution). Consequently if $K>8 / \rho$ where $\rho=T A^{2} /$ $\sigma^{2}$, the first two terms in Eq. A2 are significantly positive and dominate the other $\left(\sigma^{2} / 2 T\right) \chi_{2}^{2}$ spectrum levels for $\kappa \neq \kappa_{0}$, and thus the maximum of the frequency-wavenumber spectrum will occur at a $\kappa$ near $\kappa_{0}$, i. e., $\hat{\kappa} \approx \kappa_{0}$. The error in $\kappa$ will then be due to the interpolation error resulting from the finiteness of the aperture. In other words, if $K>8 \rho^{-1}$, the array has sufficient redundancy to resolve the signal given the signal-to-noise level.

*Present address: Sherman Fairchild Distinguished Scholar, California Institute of Technology, Pasadena, CA 91125.

${ }^{1}$ M. J. Hinich and P. Shaman, "Parameter Estimation for an $r$-Dimensional Plane Wave Observed with Additive Independent Gausian Errors," Ann. Math. Stat. 43, 153-169 (1972).

${ }^{2}$ C. S. Clay, M. J. Hinich, and P. Shaman, "Error Analysis of Velocity and Direction Measurements of Plane Waves Using Thick Large-Aperture Arrays," J. Acoust. Soc. Am. 53, 1161-1166 (1973).

${ }^{3} \mathrm{~J}$. Capon and P. Green, "Statistical Accuracy of Data Used in Seismic Inversion," Geophys. J. R. Astron. Soc. 21, 373386 (1970).

${ }^{4}$ M. J. Levin, "Least-Squares Array Processing for Signals of Unknown Form," Radio Electron. Eng. 29, 213-222 (1965).

${ }^{5} \mathrm{H}$. Cramér, Mathematical Methods of Statistics (Princeton U. P., Princeton, NJ, 1946).

${ }^{6}$ E. F. Fama and R. Roll, "Parametric Estimates for Symmetric Stable Distributions," J. Am. Stat. Assoc. 66, 331-338 (1971).

${ }^{7}$ G. P. Box and M. E. Muller, "A Note on the Generation of Random Normal Deviates," Ann. Math. Stat. 29, 610-611 (1958).

${ }^{8}$ Random Number Generation and Testing IBM C20-8011. 